\title{
Location-Based Services
}

\section{Huynh Tan Hoi ${ }^{*}$, Le Vu Truong ${ }^{2}$}

${ }^{1}$ Japanese Department, FPT University, Ho Chi Minh City, VIETNAM

${ }^{2}$ Department of IT, FPT University, Ho Chi Minh City, VIETNAM

*Corresponding Contact:

Email: hoiht@fpt.edu.vn

Manuscript Received: 15 July 2019 - Revised: 07 Sept 2019 - Accepted: 12 Oct 2019

\begin{abstract}
In the current information technology trend, GIS (Geographic Information Systems) and GPS (Global Positioning Systems) are potential systems providing convenient, valuable services. They are a fundamental base in location-based services (LBS). LBS is an object positioning and monitoring system with a major combination of advanced technologies such as mobile communications, GPS technology, GIS and the Internet. The article consists of two sections in which the overview of the LBS system is presented first, followed by the introduction of the LBS system structure used to build the VTS - Vehicle Tracking System at Dong A Bank.
\end{abstract}

Keywords: GIS, Information Technology, LBS, Potential Systems

This article is is licensed under a Creative Commons Attribution-NonCommercial 4.0 International License. Attribution-NonCommercial (CC BY-NC) license lets others remix, tweak, and build upon work non-commercially, and although the new works must also acknowledge \& be non-commercial.

\section{INTRODUCTION}

The application of Location-Based Services (LBS) in establishing vehicle tracking system at Dong A Bank. Location-based service (LBS) terminology is a recent concept signifying applications that integrate geographic location (i.e., spatial coordinates) with the general conceptions of services. Typical examples of these applications are rescue services, vehicle navigation systems, travel planning, and location and information search services which are similar to the combination of Yellow Pages and electronic maps. Thanks to the development of mobile communications, these applications represent a new challenge of both conceptual and technical aspects. Obviously, most will be playing an important role as a part of everyday life in the future. They operate on computers, PDAs (personal digital assistants), mobile phones, etc. so as to provide value-added services of location information for the users. LBS is defined as "services that integrate the location of a mobile device or location with other information to provide added values for the users." (Schiller \& Voisard, 2004).

\section{The Generalization OF LBS SYSTEM}

Major components of the LBS system include mobile devices, communications networks, navigation systems, and the providers of applications and services as well as contents and data. 


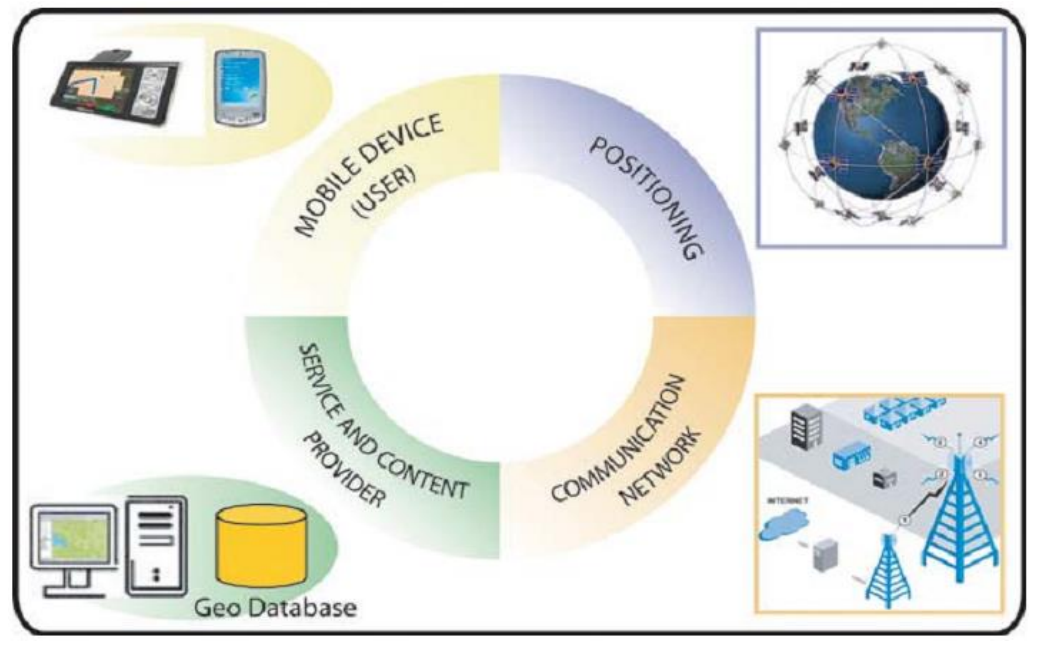

Figure: Components of the LBS system

Mobile devices which can be cell phones, PDAs, laptops, car navigation devices and information providing stations in public places are used to send requests and receive corresponding results. The positioning system is responsible for providing client location data as an input parameter for the processing requests. Specifically, the service providers need to know the clients' current location to deliver accurate guidance in navigational systems or to gain access to clients as quickly as possible with services in terms of technical supports, health and goods delivery.

In some other cases, clients' locations are the basic used by the providers for charging their services costs. In fact, the GPS - Global Positioning System is the prior choice when planning to set up LBS systems. Due to dense degree of coverage area on earth with high accuracy and the access to use free signals, most mobile devices have built-in GPS positioning functions. Communication networks play the role of connecting mobile devices and service providers. In case the service providers deploy their applications on the Internet, the communication networks are understood as mobile information networks and the Internet. During operating process, clients' requests are sent to the interface between the cellular network and the Internet via cellular network, from which requests are forwarded to the service providers.

The application and service providers receive requests from the clients, locate them through location data attached to the requests, and then process them to provide assistance or information corresponding to the locations and requirements of the clients. Applications and services deployed by the providers are usually navigational aids, geographic reference, rescue, vehicles monitoring.

In fact, most LBS systems are modeled as service providers and content providers' model. However, service providers occasionally do not own the data source. They must take them from the content providers instead. Each content provider usually possesses data warehouses of different areas, for example, geographic data, traffic data, financial system data, etc. Ordinary users are unnecessary to pay attention to the existence of the content providers as we do not work directly with them. The service and application providers are in charge of it. 


\section{TYPES OF LBS SYSTEMS}

Today, LBS systems are being applied in a lot of fields to serve different needs. In general, they can be divided into four main categories: Tracking Services, Emergency Services, and Location Based Information Services) and Location Sensitive Billing.

\section{Management and supervision services}

First, monitoring devices are attached to vehicles such as cars, motorcycles, laptops, etc. Then, such devices automatically send their current location signals to the data center of the service providers if they are activated. Once the position data is assembled, the route of the device is drawn on its map. As a result, the current location of the object being monitored is accurately identified in order to reach the object as needed, for example, to find the lost object. Management and supervision services are also applied to some other management and operation systems of taxi, fleet, cash etc., for instance. In addition to benefit the users, the position data is used to assist the operator in making rational decisions for improving the performance of the whole systems.

\section{Urgent support services}

In social and daily activities like taking part in traffic, tourism and exploration, we sometimes face dangerous situations by accident. That a timely hand comes for aids is extremely necessary to get high safety. The emergency support system operates on the principle that the service providers receive the assistance requests from the clients, then they will send technical equipment and necessary manpower to the location at which the client's coordinates have been detected so as to provide supports opportunely. However, the service users need equipping with a device that allows them to easily send the required signals when needed and provide accurate location information so as to assist the facilitators in reaching the place expeditiously.

\section{Services provide location-based information}

Imagine you are driving to work and suddenly you notice your car is running out of petrol. Immediately, the first thing you may think of is to fill the fuel at the nearest petrol station. Unfortunately, neither your cash nor the ATM is available. In this situation, you can use your smart handset such as a smartphone, a PDA, or a tablet which can be fixed onto your vehicle and supports GPS and wireless 3G or GPRS Internet connectivity, then access a location service on the device. Depending on types of the devices you use and the operating principles of the service providing system, the service accessing process can be conducted in one of two ways:

First, if the service is deployed by the provider as a Wireless Application Protocol (WAP), then the clients can connect to the system via web browsers regularly integrated in the devices' operating system (OS). The websites the clients go to will provide a graphical interface allowing them to send the requested content to the service providers. After having been added with the location data, the requested content will be expressed in HTML format and sent via the Hypertext Transfer Protocol (HTTP). The web application receives and processes the requests and then response results to the service users in the same format and protocol they used to send the requests. The process of a request at Web Server takes place in the following order:

Step 1: Receive and analyze the request. Location data is obtained from which the request is sent.

Step 2: Based on the location data to determine the current user's coordinates on the map.

Step 3: Search for the desired objects (ATM, gas station) and select the one closest to the user location. 
Step 4: Find and mark routes from the current client's location to the identified place of the objects.

Step 5: Send the navigation map to the client as an image file.

In order to facilitate the processing of Geographic Information System (GIS) data, the digital map set in the web server is built up as vector model, in which each object in the map is formed of basic elements including points, lines, polylines and regions. Continuously, the web server will capture the vector map and then obtain it as a raster map before viewing it on the handsets. Indeed, the raster map is an image file where each pixel is associated with a coordinate in the geographic coordinates (latitude, longitude). To speed up transferring data, the raster map is usually compressed to JPEG or GIF format before being sent to the client (Ahson, \& Ilyas, 2011).

Secondly, the system is deployed in Client-Server model. A Client-Server modeled consists of two components, the client component installed on the client device, the server component installed on the computing system of the service provider. In this model, the client component plays the role similar to that of the web browser. The difference between the two is in the system operating principle. The first one is, the Client software is integrated a base map (possibly in the form of vector or raster). Because of memory capacity limitation and processing speed, the geographic information database of the Client software is limited in size. Therefore, the background map attached to it will be very simple, probably just like the road network. The second one is, as the client software itself has a base map, so in the step, the server software only needs to return the data bytes in character (much less compact than sending a map in the form image file). The Client software will decode the data bytes to display the location of the object (ATM, gas station) and the path to go on the base map (Grewal, Weill \& Andrews, 2001).

Comparing these two models, the Client-Server model has the advantage of fast response rates due to the reduction of data size sent from the supplier to the client but it requires clients to install a part Soft on their device.

\section{Location-based billing service}

Some systems need to determine the location of the customer to charge for the service they will provide. For example, the remote purchase service, shipping service ... So, location billing is actually a function of a system, this function helps to calculate the cost of services more accurately through This makes the customer more confident in the system (Küpper, 2005).

\section{Building a Vehicle Monitoring System at Dong A Bank}

With its strong development, Dong A Bank has a large network and covers almost all provinces and cities in Vietnam with more than 200 branches / transaction offices and more than 1200 cash withdrawal points. ATM / POS transactions. Along with that growth, the number of means of service, transportation (money, documents, equipment ...) between units and cash for ATMs also increased. Therefore, the management, coordination and supervision of the means at the bank is a very difficult task, spending money and manpower. The current management of the bank has many shortcomings such as: Managers can't know the current location, route, amount of fuel consumed, travel time of each vehicle; It is impossible to know the exact location of the vehicle in time for emergencies (means of damage along the road, traffic accidents, robberies ...).

In order to increase the ability to manage and monitor vehicles and to minimize the risks in the management and operation process, the requirement for a Vehicle Tracking System (VTS) has been established. With the construction of VTS under the LBS model, information about transportation vehicles such as current coordinates, speed of travel, transportation routes, and fuel consumption will be updated to the control center. This database is based on this database. 
The operating center can calculate the fuel of each transport vehicle, check the incident or receive emergency status reports (SOS) during the circulation of the means, optimizing the management and operation.

\section{Select a Deployment Plan}

The option chosen is to build an online media monitoring system that is between the operating center and the online communication medium, exchanging data with each other. In this method we need the following means and systems:

At the control center

- The map displays the number of each geographic area.

- Servers and workstations according to management needs with GIS management information system using digital map management software (MapInfo, Goggle map ...).

On board of transport

- $\quad$ GPS module.

- GPS data collection and transmission via GPRS, 3G wireless Internet.

Data sent from the device to the hub includes the device ID and full location information in a unified standard.

Principles of system operation

The medium and medium communication channel can operate in one of the following modes:

- Maintaining a seamless connection: The connection is always ready for data transfer on location and time.

- Automatic connection setup: The communication channel is automatically set when either party has the data to transmit.

- Manually establish a connection: The operator or operator of the medium performs a link initiation on its own when sending a message.

Equip equipment and build management program using GPS technology

The device is mounted on the vehicle is a GPS module with display screen and can access wireless Internet (GPRS, 3G ...) or can use smart phones, PDA with integrated GPS, GPRS / 3G. Equipment must ensure the following information:

- The latitude and longitude coordinates of the means and the height of the sea surface.

- The current time is GMT.

- $\quad$ The speed and direction of movement of the means.

- Number of satellites receiving the signal.

The above parameters are packaged into standard messages and sent to the center.

The management system at the control center functions to update the data received from the GPS receiver on the means of transport, integrate with the digital map to do the task:

- Updating, displaying the monitored vehicles online on the command screen.

- Notification of the status of means of transport.

- Providing notices on fuel, operating time of each means.

- Monitor incidents and receive emergency situations from the means of transportation. 
- Supports analysis and identification of time-saving routes from the starting point to the destination of each vehicle and allows the on-screen display of equipment mounted on the vehicle.

With these facilities and management information systems, the vehicle monitoring process will be as follows:

- Vehicle activation for GPS device operation.

- Select the desired location on the list

- The system will rely on the current location of the Vehicle and the location of the destination to find the most time-saving route.

- The means of receiving and displaying the itinerary as recommended by the system.

- The media will start to arrive and arrive at the destination.

- During the process of moving the GPS data on a postal vehicle, it is sent continuously to the center via the wireless Internet according to the prescribed standards.

- The center receives data and displays on the command screen using the GIS Management Information System. When needed the center may request some information from the postal vehicle.

- Update the data sent to save as the management file in the center.

The construction system has met the demand and has made management, monitoring and operation simpler, easier and more cost-effective.

\section{CONCLUSION}

This report presents an overview of Location Based Services (LBS) and briefly discusses the application of LBS to build a vehicle surveillance system in East Asia Bank. The paper presents the options and options for GPS data acquisition devices, as well as how to set up a management information system at the control center. Currently, the author is directly involved in building this system. Although there are some difficulties in the implementation process, but with the preliminary findings above, the construction of a vehicle monitoring system at Dong A Bank is entirely feasible and effective. Highly compatible with modern management technologies. In addition, this is a high value-added mobile network-based business that applies GPS technology to manage and provide roadside services, positioning, incident reporting. To report on repair and emergency rescue stations for traffic means on the road.

\section{REFERENCES}

Ahson, Syed A., \& Ilyas, Mohammad. (2011). Location Based Services Handbook. New York: CRC Press.

Grewal, M. S., Weill, L. R., \& Andrews, A. P. (2001). GPS: Intertial Navigation and Intergration.New York: John Wiley \& Sons Inc.

Küpper, A. (2005). Location-based Services: Fundamentals and Operation. Ludwig Maximilian University Munich, Germany: John Wiley \& Sons Ltd.

Schiller, J., \& Voisard. A. (2004). Location-Based Services. San Francisco: Morgan Kaufmann Publishers.

$$
--0--
$$

